\title{
Proportional-Fair Multi-User Scalable Layered Wireless Video Streaming Powered by Energy Harvesting
}

\author{
Jian Yang, Senior Member, IEEE, Jinsen Xie, Shuangwu Chen, Zilei Wang, Member, IEEE, Han Hu, \\ Member, IEEE and Lajos Hanzo, Fellow, IEEE
}

\begin{abstract}
The problem of adaptive multi-user scalable layered video transmission is considered in energy harvesting (EH) aided wireless communication systems. With the goal of improving the quality of video services while providing fairness amongst the users despite the random nature of both energy harvesting and the channel quality, we formulate our Scalable Video Coding (SVC) design as a Constrained Utility Function Maximization (CUFM) problem. The proportional fairness and playback smoothness of our design is guaranteed by maximizing the log-sum of the users' video qualities, while satisfying the battery fullness constraint and video layer (quality) fluctuation constraint. By invoking the classical Lyapunov drift based optimization technique, we further decompose the CUFM problem into two parallel subproblems, i.e., a dynamic transmission power allocation problem and a dynamic layer selection problem. By solving these two subproblems, we derive a joint power allocation and video layer selection strategy for multi-user SVC video transmission. The theoretical performance bound of the proposed solution is also presented. Numerical simulations are conducted with real H.264 SVC video traces and the experimental results demonstrate the reduced playback interruption rate and layer switching rate compared to a heuristic algorithm ProNTO. The results also illustrate a tradeoff between the system's utility function and the playback smoothness experienced by the users.
\end{abstract}

Keywords-Energy harvesting, proportional fairness, energyefficient communication system, scalable video coding, Lyapunov optimization, feasible direction method.

\section{INTRODUCTION}

$\mathbf{E}$ NERGY Harvesting (EH) constitutes a promising green and self-sustainable communication technique, where apart from data transmission, the nodes also accumulate energy from the renewable sources like mechanical vibration, solar, pressure and electromagentic radiation, thus reducing the use

J. Yang, J. Xie, S. Chen, and Z. Wang are with the School of Information Science and Technology, Univ. of Science and Technology of China, Hefei 230027, Anhui, China. E-mail:jianyang@ustc.edu.cn, jasenxie@mail.ustc.edu.cn, chensw@ustc.edu.cn, zlwang@ustc.edu.cn

$\mathrm{H}$. $\mathrm{Hu}$ is with the Beijing Institute of Technology, Beijing, China. Email:hhu@bit.edu.cn

L. Hanzo is with the Department of Electronics and Computer Science, University of Southampton, SO17 1BJ, UK. E-mail:1h@ecs.soton.ac.uk.

L. Hanzo would like to acknowledge the financial support of the Engineering and Physical Sciences Research Council projects EP/Noo4558/1, EP/PO34284/1, COALESCE, of the Royal Society's Global Challenges Research Fund Grant as well as of the European Research Council's Advanced Fellow Grant QuantCom. of fossil fuels. IDTechEx predicts that the energy harvesting market grows to $\$ 2.6$ billion by 2024 [1]. Internet of Things (IoT) applications and devices are capable of simplifying human activities, and $\mathrm{EH}$ has received significant research attention for extending their battery life [2], [3].

Video streaming has been widely deployed in various network applications and services [4]-[7]. According to the Cisco's white paper [8], by 2021 video traffic will constitute $82 \%$ of all the global Internet traffic. Energy harvesting is also expected to support real-time video streaming services. Although the power efficient transmission problem of $\mathrm{EH}$ aided communications system has indeed been richly characterized in the literature [9]-[13], throughput maximization has been the main focus in these treatises, whilst dedicating little attention to the upper-layer applications. Explicitly, throughput maximization does not lead to Quality-of-Experience (QoE) optimization owing to the resultant excessive packet-loss events, especially not in multiuser scenarios. This motivates us to conceive a new transmission strategy for multi-user video streaming in $\mathrm{EH}$ aided communication systems.

Scalable Video Coding (SVC) has been emerging as an advanced video compression technique [14] supporting onthe-fly bitrate adaptation for video streaming, which breaks a single-stream video into a multi-stream flow in terms of a base layer and a few enhancement layers. This technique enables the receiver/sender to most appropriately choose the number of transmitted video layers for achieving the required video quality, depending on the prevalent conditions of both the network and of the device itself. The capability of onthe-fly bitrate adaptation provided by SVC motivates us to adopt it for accommodating the stochastically time-varying nature of both the energy harvesting process and of the channel quality, thus supporting resilient video transmission in $\mathrm{EH}-$ aided wireless communication systems. The intuitive principle of this is that for the scenario of low harvested energy only the video base layer is streamed in order to keep a low likelihood of playback interruptions, which would seriously degrade the quality perception of the users, while for the scenario of having abundant harvested energy the streaming of more enhancement layers is desired for achieving a higher video quality. Hence, our paper focuses on the problem of the optimal allocation of stochastically harvested energy for scalable video transmission. This problem becomes more complex in the scenario of multiuser video transmission due to their different channel qualities at the receivers and the requirement of maintaining fairness 
in terms of the received video quality of all the users. To the best of our knowledge, the multi-user video streaming problem of EH aided wireless communication systems has not been explored in the open literature.

One potential promising application of EH aided multi-user SVC streaming is satellite-5G network typically powered by harvested solar energy [15], which is depicted in Fig. 1. The SVC video sequences are uploaded from the ground station to the communication satellite, and then from the satellite to the moving vehicles. In contrast to the traditional ground-cell network, the coverage of the communication satellite is wide, which can help reduce the handover frequency of the terrestrial vehicles and is conducive to providing seamless networking services [16]. In energy harvesting communication systems, the proposed methods that mainly focus on maximizing the throughput are not directly applicable to video transmission optimization. Our early work [17], [18] have solved the SVC video transmission problem in the single-user scenario over energy harvesting systems. However, in multi-user video transmission scenario, which can be found in satellite-5G network, the communication systems will be confronted with new difficulties and challenges, such as the fairness guarantee among the users and dimensional disaster caused by multiuser. All of these motivate us to derive a new strategy that can solve the multi-user video transmission problem in energy harvesting communication systems.

This paper focuses on the problem of downlink adaptive scalable video streaming in multi-user $\mathrm{EH}$-aided wireless communication systems. Explicitly, we jointly consider the energy resource allocation and the fairness among the users. In our problem formulation, we conceive a total utility function in terms of the log-sum of the video qualities, i.e., Peak Signalto-Noise Ratio (PSNR), for the sake of achieving proportional fairness. We further conceive an online Power Allocation and Layer Selection (PALS) strategy for improving the transmitted video quality subject to a maximum tolerable playback interruption rate. Our contributions are listed as follows:

- This paper studies the multi-user SVC video transmission problem of energy harvesting communication systems. Considering the characteristics of the SVC video and the fairness among the users, the multi-user video transmission problem is formulated as a Constrained Utility Function Maximization (CUFM) problem, where the log-sum of the PSNR is chosen as the optimization objective for guaranteeing proportional fairness.

- We consider the energy buffer fullness constraint and video quality fluctuation constraint for the sake of maintaining a smoothened perception of the video playback quality. This implies that our problem formulation maximizes the overall video quality subject to the constraints of proportional fairness and smoothened video quality.

- We introduce the classical Lyapunov stochastic optimization theory to transform the original CUFM problem into a Lyapunov optimization problem, which can be further decomposed into two independent sub-problems, i.e., the energy allocation and the video layer selection problems. Unlike the layer-traversal algorithm proposed in [17], whose computational complexity is on the order

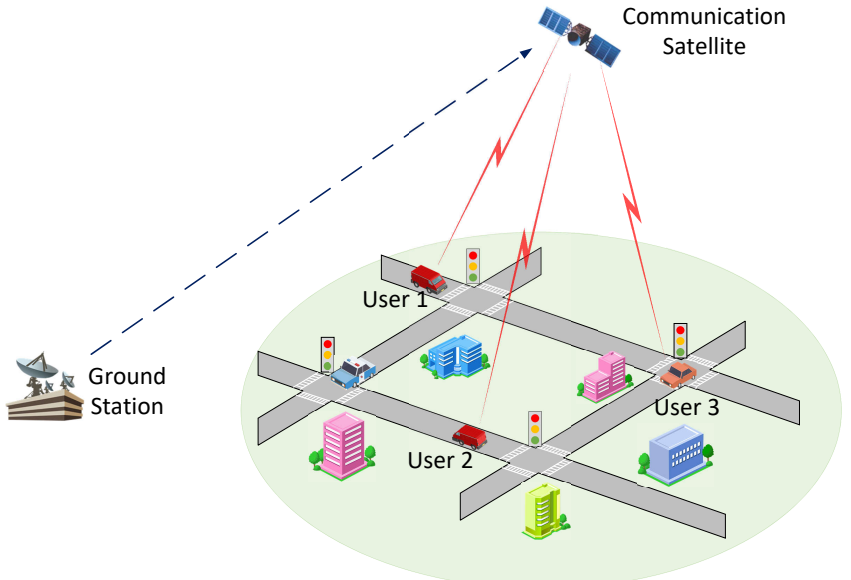

Fig. 1: EH aided multi-user SVC streaming application in satellite-5G network.

of $O\left(L^{K}\right)$ in a K-user system, we rely on the feasible direction method of [19] to solve the video layer selection problem and thus, the computational complexity is reduced to the order of $O(K L)$.

- Real video traces are utilized to conduct experiments for demonstrating the efficiency of the proposed PALS strategy. A benchmark algorithm termed as ProNTO [20], which was proposed for maximizing the throughput of energy harvesting systems, is used for our performance comparisons. Furthermore, the performance sensitivity to the parameters of our solution is also investigated for fine-tuning the parameter settings.

The rest of the paper is organized as follows. Section II discusses the related work. Section III is devoted to formulating the CUFM problem taking into account both the harvested energy and the wireless channel, while Section IV elaborates on the derivation of the online PALS strategy for solving the CUFM problem. Our simulation results characterizing PALS are presented in Section V. Finally, we conclude in Section VI.

\section{RELATED WORK}

\section{A. Energy Harvesting Wireless Communication Systems}

Recently, energy harvesting communication systems have received significant research attentions [10]-[13], [21]-[33]. In [21], wireless networks capable of harvesting RF energy were analyzed while the throughput maximization problem of $\mathrm{EH}$ systems was studied in [10]-[12]. A two-stage water filling policy was derived in [23] for the throughput maximization of a communication system that is powered both by harvested energy and by the power grid. The authors of [24] proposed an optimal packet scheduling policy for dynamically adjusting the transmission rate according to the energy and the traffic load of an EH communication system. Since most of the devices in IoT are low-power battery fed systems, the finite charge of such devices brings about new challenges in terms of system 
deployment and data transmission. To tackle this problem, a novel Integrated Information Relay and Energy Supply based RF harvesting model was proposed in [25]. A throughput constrained problem dedicated to energy efficiency and transmission rate maximization in RF-EH communication systems was studied in [26]. The authors of [27] formulated the power vs delay tradeoff as a stochastic problem, which is solved by Lyapunov optimization theory. By jointly considering the power allocation and time scheduling policy, an algorithm was proposed in [28] to solve the end-to-end throughput maximization problem in two-hop-relaying wireless systems, where the relay node is capable of harvesting energy. In [13], the per-flow performance of an EH multichannel system was investigated.

Since communication systems equipped with $\mathrm{EH}$ constitute a new video streaming scenario [17], optimizing the QoE at the receivers constitutes an interesting new research problem. The solutions discussed in the aforementioned contributions mainly focused on improving the energy efficiency or the throughput, without giving any cognizance to the applicationlevel perceptual video quality. Hence, they are not directly applicable to video transmission optimization in EH communication systems.

\section{B. Adaptive Scalable Video Transmission With Sufficient En- ergy}

SVC constitutes an extended version of H.264/AVC, designed for real-time video transmission and playback under diverse conditions. Resource scheduling strategies and transmission algorithms have been widely investigated in the literature [17], [18], [34]-[42], and a comprehensive overview of SVC-based video characteristics can be found in [14], [43], [44]. In [14], a trace-based evaluation method was introduced for SVC video streaming systems. The QoE-aware SVC video transmission problem and a sophisticated power allocation scheme was designed for Multi-Input Multi-Output (MIMO) systems in [34] and [35]. The authors of [36] jointly configured both the power and the transmission rate with the objective of maximizing the decoding quality of SVC video streaming in MIMO systems. As a further development, a cross-layer optimization architecture was designed in [37] for scalable video delivery over OFDMA wireless networks. Both [38] and [39] have investigated the adaptive layer switching algorithm and resource allocation problem for SVC video streaming in wireless communication networks. Furthermore, by grouping the moving vehicles into cooperative clusters, novel V2V (vehicle-to-vehicle) communication techniques were proposed in [40] for real-time SVC video streaming in vehicular networks. In [41], a novel SVC-based multi-user transmission policy was proposed for wireless networks having sufficient energy supply. In [42], a joint video rate and power control framework was proposed for multi-node wireless networks in order to satisfy the delay constraint associated with the application-level data characteristics. However, the aforementioned scalable video streaming strategies are mainly discussed in the scenarios where the energy is always sufficient. Hence they are not directly applicable to EH-aided wireless scalable video streaming systems, where the amount of harvested energy is not considered.

\section{SVC Video Transmission in EH-aided Communication Sys- tems}

In contrast to wireless SVC video streaming relying on the mains power supply, there is a paucity of adaptive techniques designed for EH aided wireless video transmission. In [17] and [18], the SVC video transmission was discussed in EH wireless communication systems in a single-user scenario. In [18], an energy starvation probability estimation model relying on the Large Deviation Principle (LDP) was proposed, which was used for adjusting the number of video layers transmitted in a single-user scenario. The authors of [17] derived a dynamic layer selection aided transmission algorithm (DLTA) conceived for single-user EH aided wireless SVC streaming. But again, EH aided multi-user video streaming, routinely found in satellite-5G networks powered by harvested solar energy [15], has only received very limited attention.

\section{PROBLEM FORMULATION}

We consider an EH wireless communication system having $K$ users served by a wireless transmitter which is equipped with a rechargeable battery, as depicted in Fig. ??. The battery is charged by an energy harvester, gleaning ambient energy on board the satellite from solar radiation. We divide the time into uniform slots of size $d$. Each frame of the SVC videos consists of $L$ layers, a base layer and $(L-1)$ enhancement layers. The number of the video layers to be transmitted are determined by the decision device, subject to different wireless channel conditions of the $K$ users and the amount of energy stored in the battery. Let $\gamma_{k, n}, k \in \mathcal{K} \triangleq\{1,2, \ldots K\}$, denote the channel gain of the link between the transmitter and user $k$ at time slot $n$, during which the fading conditions are assumed to remain constant, but may change across the time slots. Here, we consider Time-Division Multiple Access (TDMA) for multiple users to share the same wireless channel having a frequency bandwidth of $W$. For the sake of achieving realtime video streaming, the SVC video packets queued at the transmitter have to be transmitted to all the users immediately during the next time slot. If the energy remaining in the battery is insufficient, the video packets are discarded, which will cause playback interruptions.

\section{A. Wireless Channel Model}

Fig. 3. shows the transmission model of the wireless channels between the transmitter and the $K$ users. The video bits are transmitted to the $K$ users in a time-switching manner due to the TDMA regime employed. During time slot $n$ the bits transmitted to user $k$ have to obey Shannon's formula of

$$
D_{k, n}=t_{k, n} W \log _{2}\left(1+\gamma_{k, n} \frac{P_{n}^{T}}{N_{o} W}\right) \text {, }
$$

where $t_{k, n}$ and $N_{o}$ represent the transmission time of user $k$ and the power of the additive white Gaussian noise (AWGN) [18]. Further more, we have $\sum_{k=1}^{K} t_{k, n} \leqslant d$ due to the duration constraint of the time slots. And $P_{n}^{T}$ is the transmission power in the $n$th time slot. 


\section{B. Model for Rechargeable Battery}

As shown in Fig. ??, the energy is harvested from the environment and stored in a rechargeable battery having a maximum capacity of $B_{\max }$. Let $E_{n}^{T}$ and $E_{n}^{H}$ represent the energy consumed by video transmission and harvested during the slot $n$, respectively. It was assumed in [17] and [45] that the harvested energy packet $E_{n}^{H}$ can be considered as an i.i.d. variable. At the start of slot $n$, the remaining energy of the transmitter is $B_{n}$. We assume that the harvested energy $E_{n}^{H}$ is available until the beginning of slot $n+1$. Then, the update formula of the energy stored in the battery can be written as follows:

$$
B_{n+1}=\min \left\{\max \left\{B_{n}-E_{n}^{T}, 0\right\}+E_{n}^{H}, B_{\max }\right\} .
$$

The transmission power, determined by the Decision Device, is allowed to vary across time slots, but it is kept constant during each slot. Thus, the energy used for video transmission is

$$
E_{n}^{T}=P_{n}^{T} d,
$$

where $P_{n}^{T}$ denotes the transmission power. In video services, users tend to prefer a reduced video quality over playback interruptions, since playback interruptions seriously reduce the quality of experience. In an EH communication system, if the energy is depleted, no video can be transmitted, thus imposing a playback interruption. Therefore, in order to avoid playback interruptions, it is necessary to reserve a certain amount of energy at the transmitter for delivering the forthcoming packets of the video base layers.

The long-term time-averaged value of the battery level is defined as $\bar{B} \triangleq \lim _{N \rightarrow \infty} \frac{1}{N} \sum_{n=1}^{N} B_{n}\left(P_{n}^{T}\right)$. A certain amount of energy should be maintained for supporting uninterrupted video streaming, and thus avoiding QoE degradation for the end users. In order to achieve this, the time-average $\bar{B}$ is constrained by a pre-defined energy threshold $\theta$ :

$$
\bar{B} \geqslant \theta \text {. }
$$

Since higher video quality requires more video layers, this is more likely to exhaust the battery in the absence of any energy reservation. Once the battery energy is exhausted, the forthcoming video packets will be discarded and the QoE of the end users will be severely degraded. Hence, we apply the constraint (4) to achieve a sustainable video transmission.

\section{Video Quality Fluctuation}

Apart from video playback interruption, fluctuations in video quality constitute another factor affecting the user experience in video applications. Varying the number of video layers frequently will substantially reduce the end user's QoE. The difference of the transmitted layers between two adjacent time slots is used for characterizing the fluctuation of the video quality, i.e., $f_{k, n}=\left|x_{k, n}-x_{k, n-1}\right|$, where $x_{k, n}$ is the number of transmitted video layers corresponding to user $k$ at the time slot $n$, and $f_{k, n} \in \mathcal{F} \triangleq\{0, \ldots, L-1\}$. The time-averaged layer fluctuation $\overline{f_{k, n}}$ is defined as $\overline{f_{k, n}} \triangleq \lim _{N \rightarrow \infty} \frac{1}{N} \sum_{n=1}^{N} f_{k, n}$. In order to achieve a smoothly evolving video quality, the average layer fluctuation should be kept below a certain

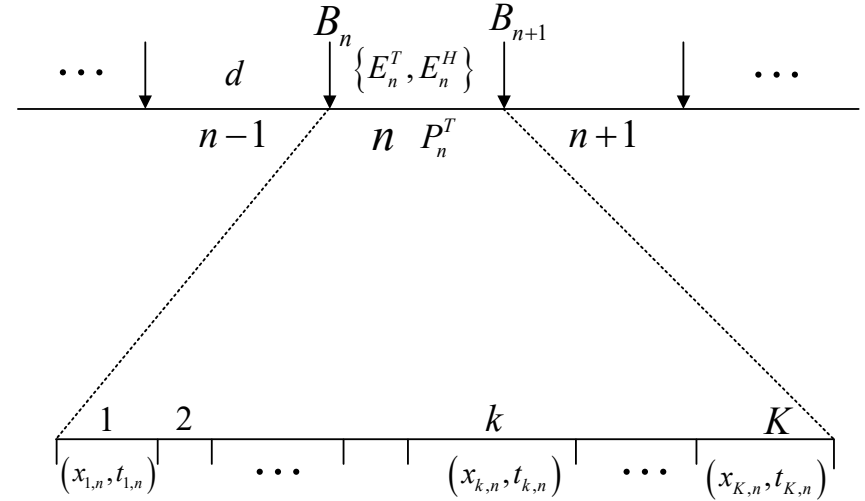

Fig. 3: Transmission model, the time of the $n$-th slot is allocated to $K$ users for video data transmission.

threshold $\eta$. Then we have a constraint imposed on the timeaveraged fluctuation as

$$
\overline{f_{k, n}} \leqslant \eta .
$$

Here, we use the layer switching frequency as a QoE metric for characterizing the video quality fluctuation. However, according to [17] the PSNR mainly depends on the base layer, while the contribution of each enhancement layer is similar, which implies that the video layer switching frequency is a reasonable metric of characterizing the video quality fluctuation.

\section{Video Quality Maximization Problem Formulation to Guar- antee Proportional Fairness}

The authors of [20], [46] have demonstrated that proportional fairness among the users may be guaranteed in terms of the log-sum based system utility. Inspired by this work, the system utility in our EH-aided multi-user communication system is defined as the log-sum of all the users video quality ${ }^{1}$ as follows:

$$
U_{n}=\sum_{k=1}^{K} \log _{2}\left(1+P S N R_{k, n}\right),
$$

where $P S N R_{k, n}$ (Peak Signal-to-Noise Ratio) characterizes the received video quality of user $k$ during the $n$-th time slot, which is related to the transmitted video layers. Then, we formulate the optimization problem constrained by playback smoothness and energy dissipation. At the $n$th time slot, the Decision Device of the transmitter embarks on transmitting $X_{n}=\left(x_{1, n}, \ldots, x_{k, n}, \ldots, x_{K, n}\right)$ layers to the users, where $x_{k, n} \in \mathcal{X} \triangleq\{0, \ldots, L\}$, and $x_{k, n}=0$ means that no video layer is transmitted to user $k$. In order to achieve low-delay video transmission, the dropped video packets will not be retransmitted. Once the base layer is discarded due to having insufficient energy, playback interruptions occur for all the users during an entire time slot, which severely reduces the

\footnotetext{
${ }^{1}$ The defined utility function is strictly convex, and plus one can avoid negative infinity when $P S N R_{k, n}=0$.
} 
perceived video quality. For this reason, constraint (4) is introduced to keep a certain amount of energy in the battery for near-future base layer transmission. Let $Q_{k, n}=\varphi\left(x_{k, n}, P_{n}^{T}\right)$ denote the near-instantaneous PSNR value achieved by user $k$ upon receiving $x_{k, n}$ video layers, when the transmission power is $P_{n}^{T}$. Then, the log-sum video quality metric of (6) used for guaranteeing proportional fairness can be rewritten as $U_{n}\left(X_{n}, P_{n}^{T}\right)=\sum_{i=k}^{K} \log _{2}\left(1+Q_{k, n}\right)$. Accordingly, the timeaveraged total video quality constituting our utility function is defined as:

$$
\bar{U}=\lim _{N \rightarrow \infty} \frac{1}{N} \sum_{n=1}^{N} U_{n}\left(X_{n}, P_{n}^{T}\right) .
$$

Since we aim for maximizing the utility function in (7), while the constraints (4) and (5) are both satisfied, our constrained utility function maximization (CUFM) problem associated with proportional fairness is formulated as:

$$
\begin{array}{cl}
\text { Maximize } & \bar{U} \\
\text { Subject to } & \bar{B} \geqslant \theta, \\
& \overline{f_{k, n}} \leqslant \eta, \quad k=1,2, \ldots, K \\
& \sum_{k=1}^{K} t_{k, n} \leqslant d, \\
\text { Action } \quad & \left\{P_{n}^{T}, X_{n}\right\} .
\end{array}
$$

In order to solve the above problem, the wireless transmitter has to make decisions both about the optimal transmission power and the number of video layers to transmit at each time slot.

\section{DYNAMIC POWER ALLOCATION AND VIDEO LAYER SELECTION STRATEGY}

In this section, the Lyapunov optimization theory [47] will be used for solving problem (8), and a dynamic online strategy is derived for determining the optimal number of transmission layers and the power allocation. In Section IV-C, the performance bound of the proposed method is analyzed theoretically.

\section{A. Lyapunov Stochastic Optimization Formulation}

For the sake of solving the CUFM problem via the classical Lyapunov optimization technique, $K+1$ virtual queues $\left(H_{n}\right.$ and $\left.z_{k, n}, k \in\{1,2, \ldots, K\}\right)$ are constructed for transforming the constraints (4) and (5) into characterizing the queue stability. According to the theory of Lyapunov optimization [47], the dynamics of the virtual queues are characterized as follows:

$$
H_{n+1}=\left\{H_{n}+\theta-B_{n+1}\right\}^{+}
$$

and

$$
z_{k, n+1}=\left\{z_{k, n}+f_{k, n}-\eta\right\}^{+},
$$

where $(x)^{+} \triangleq \max \{x, 0\}$. As shown in [47], if the mean rates of $H_{n}$ and $z_{k, n}$ are stable, the energy constraint (4) and the video quality fluctuation constraint (5) are satisfied. Let
$\Theta \triangleq\left\{H_{n}, z_{k, n}\right\},(k=1,2, \ldots, K)$ denote a cascaded vector. Then, we define a quadratic Lyapunov equation as

$$
L\left(\boldsymbol{\Theta}_{n}\right)=\frac{1}{2}\left(H_{n}^{2}+\sum_{k=1}^{n} z_{k, n}^{2}\right) .
$$

Accordingly, at the $n$th time slot, we define the conditional Lyapunov drift as:

$$
\Delta\left(\boldsymbol{\Theta}_{n}\right) \triangleq \mathbb{E}\left[L\left(\boldsymbol{\Theta}_{n+1}\right)-L\left(\boldsymbol{\Theta}_{n}\right) \mid \boldsymbol{\Theta}_{n}\right] .
$$

Minimizing (12) implies that $H_{n}$ and $z_{k, n}$ are mean rate stable on the basis of Lyapunov drift theory. Since our objective is to maximize the utility function in (7), the term "drift-pluspenalty" [47], which combines the instantaneous system utility $U\left(X_{n}\right)$ and the Lyapunov drift, is defined as:

$$
\Delta\left(\boldsymbol{\Theta}_{n}\right)-V \mathbb{E}\left\{U\left(X_{n}\right) \mid \boldsymbol{\Theta}_{n}\right\},
$$

where $V \geqslant 0$ is used as an equilibrium factor in (13) to strike the trade-off between the video quality and the readout smoothness. A high $V$ increases the system's utility and hence minimize the video distortion, but it will also increase the playback interruption rate and layer switching rate. Thus, the constrained optimization problem (8) can be solved by minimizing the "drift-plus-penalty" term at the beginning of each time slot $n$.

According to Lyapunov optimization theory [47], minimizing (13) can be achieved by minimizing the upper bound of (13). Then, the bound of (13) is reformulated to develop our dynamic video layer selection and power allocation strategy. According to (9), we have

$$
\begin{aligned}
H_{n+1}^{2} & \leqslant\left(H_{n}-B_{n+1}\right)^{2}+\theta^{2}+2 H_{n} \theta \\
& =H_{n}^{2}+B_{n+1}^{2}+\theta^{2}+2 H_{n}\left(\theta-B_{n+1}\right) .
\end{aligned}
$$

Similarly

$$
z_{k, n+1}^{2} \leqslant z_{k, n}^{2}+\eta^{2}+f_{k, n}^{2}+2 z_{k, n}\left(f_{k, n}-\eta\right) .
$$

Upon substituting (2), (14) and (15) into (13), we have

$$
\begin{aligned}
\Delta & \left(\boldsymbol{\Theta}_{n}\right)-V \mathbb{E}\left\{U\left(X_{n}\right) \mid \boldsymbol{\Theta}_{n}\right\} \\
\leqslant & C_{0}+C_{1, n}+\mathbb{E}\left\{\frac{1}{2}\left(E_{n}^{T}\right)^{2}+\left(B_{n}-H_{n}\right)\left(E_{n}^{H}-E_{n}^{T}\right) \mid \boldsymbol{\Theta}_{n}\right\} \\
& +\sum_{k=1}^{K} \mathbb{E}\left\{z_{k, n}\left(f_{k, n}-\eta\right) \mid \boldsymbol{\Theta}_{n}\right\}-V \mathbb{E}\left\{U\left(X_{n}\right) \mid \boldsymbol{\Theta}_{n}\right\} \\
= & C_{0}+C_{1, n}+\frac{1}{2}\left(E_{n}^{T}\right)^{2} \\
& +\left(B_{n}-H_{n}\right)\left(\mathbb{E}\left\{E_{n}^{H}\right\}-E_{n}^{T}\right) \\
& +\sum_{i=1}^{K} z_{k, n}\left(f_{k, n}-\eta\right)-V U\left(X_{n}\right),
\end{aligned}
$$

where $C_{0}=\frac{1}{2}\left[\theta^{2}+\sum_{k=1}^{K}\left(\eta^{2}+f_{\max }^{2}\right)+\left(E_{n, \max }^{H}\right)^{2}\right]$ and $C_{1, n}=\frac{1}{2} B_{n}^{2}+H_{n}\left(\theta-B_{n}\right)$ are a pair of constants, since $B_{n}$ and $H_{n}$ are known at the $n$th time slot. According to Lyapunov optimization theory [47], the CUFM problem associated with 
proportional fairness can be solved by minimizing the sum of the last four terms on the right side of (16), rather than minimizing (13) directly. Hence, the CUFM problem associated with proportional fairness is reformulated as

$$
\begin{aligned}
\text { Minimize: } & \frac{1}{2}\left(E_{n}^{T}\left(P_{n}^{T}\right)\right)^{2} \\
& +\left(B_{n}-H_{n}\right)\left(\overline{E_{n}^{H}}-E_{n}^{T}\left(P_{n}^{T}\right)\right) \\
& +\sum_{k=1}^{K} z_{k, n}\left(f_{k, n}-\eta\right)-V U\left(X_{n}\right) \\
\text { Subject to: } & \sum_{k=1}^{K} t_{k, n} \leqslant d \\
\text { Action: } \quad & \left\{P_{n}^{T}, X_{n}\right\},
\end{aligned}
$$

where $\overline{E_{n}^{H}}=\mathbb{E}\left\{E_{n}^{H}\right\}$. Due to the stochastic nature of $E_{n}^{H}$, and the unknown probability distribution, $\overline{E_{n}^{H}}$ cannot be calculated straightforwardly. Therefore, the historical observations are utilized for the online estimation of $\overline{E_{n}^{H}}$. The estimated value is denoted by $\hat{E}_{n}^{H}$ and the exponential smoothing (ES) model is invoked for obtaining a smoothened estimate of $\overline{E_{n}^{H}}$, which is given by:

$$
\hat{E}_{n}^{H}=\rho \hat{E}_{n-1}^{H}+(1-\rho) E_{n}^{H},
$$

where the parameter $\rho$ satisfies $0<\rho<1$. Furthermore, it was recommended by Gardner's report [48] that the reasonable range should be $[0.7,0.9]$.

The term $\left(B_{n}-H_{n}\right)\left(\overline{E_{n}^{H}}-E_{n}^{T}\left(P_{n}^{T}\right)\right)$ in (17) represents a weighted expected mismatch between $\overline{E_{n}^{H}}$ and $E_{n}^{T}\left(P_{n}^{T}\right)$. According to Equation (9), if $B_{n}$ is higher than $\theta$ in some consecutive time slots, $H_{n}$ decreases. Once we have $\left(B_{n}-\right.$ $\left.H_{n}\right)>0$, the transmission power $P_{n}^{T}$ should be increased in order to minimize $\left(\overline{E_{n}^{H}}-E_{n}^{T}\left(P_{n}^{T}\right)\right)$, consequently reducing the term $\left(B_{n}-H_{n}\right)\left(\overline{E_{n}^{H}}-E_{n}^{T}\left(P_{n}^{T}\right)\right)$. By contrast, $H_{n}$ increases while $B_{n}$ is lower than $\theta$ in some of the consecutive time slots. When $\left(B_{n}-H_{n}\right)<0$ is met, $P_{n}^{T}$ should be decreased to maximize $\left(\overline{E_{n}^{H}}-E_{n}^{T}\left(P_{n}^{T}\right)\right)$, hence, $\left(B_{n}-H_{n}\right)\left(\overline{E_{n}^{H}}-E_{n}^{T}\left(P_{n}^{T}\right)\right)$ is reduced. Similarly, the weighted difference of the video layer switching frequency and threshold $\eta$ can be presented by $z_{k, n}\left(f_{k, n}-\eta\right)$ in (17). According to (10), $z_{k, n}$ can represent the accumulated value of user $k$ 's historical layer switching rate. When $z_{k, n}$ becomes larger, the number of video layers of user $k$ is less likely to be changed for achieving reduced $f_{k, n}$, and thus to decrease the term $z_{k, n}\left(f_{k, n}-\eta\right)$. Again, the parameter $V$ is a equilibrium factor between the utility and the first two terms discussed above. A larger $V$ can be chosen to focus more on the total system utility, which is demonstrated in Section V-C3.

\section{B. Power Allocation and Layer Selection (PALS) Algorithm}

If we directly search through the legitimate solution space to find the optimal solution for (17), as in reference [17], for a $K$ user system the algorithm suffers from an excessive complexity of order $O\left(L^{K}\right)$. Fortunately, the first two items in (17) only depend on the specific selection of the transmission power $P_{n}^{T}$, while the rest of the items rely on the layer selection variable
$X_{n}$. Based on this particular structure, the optimization problem (17) can be further decomposed into two independent lowcomplexity sub-problems: the power allocation problem and layer selection problem. We also employ the feasible direction method of [19] to solve the layer selection problem and thus, the computational complexity is reduced to the order of $O(K L)$.

1) Transmission Power Decision: By minimizing the first two terms of the objective function in (17), the transmission power optimization problem is given as:

$$
\begin{aligned}
\text { Minimize: } & \frac{1}{2}\left(E_{n}^{T}\left(P_{n}^{T}\right)\right)^{2} \\
& +\left(B_{n}-H_{n}\right)\left(\overline{E_{n}^{H}}-E_{n}^{T}\left(P_{n}^{T}\right)\right), \\
\text { Action: } & P_{n}^{T},
\end{aligned}
$$

where the expression (19) is a quadratic function, and the optimal $P_{n}^{T} \geq 0$ can be obtained as follows ${ }^{2}$ :

$$
\underbrace{\max \left(\frac{B_{n}-H_{n}}{d}, 0\right)}_{\text {the optimal value of } P_{n}^{T}} .
$$

2) Video Layers Selection: Below, we derive the optimal video layer selection strategy by minimizing the last part of the objective function in (17), and thus the optimization problem is written as:

$$
\begin{aligned}
\text { Minimize: } & \sum_{k=1}^{K}[\underbrace{z_{k, n}\left(f_{k, n}-\eta\right)-V \log _{2}\left(Q_{k, n}\left(x_{k, n}\right)\right)}_{h_{k, n}\left(x_{k, n}\right)}] \\
\text { Subject to: } & \sum_{k=1}^{K} t_{k, n} \leqslant d \\
\text { Action: } & X_{n} .
\end{aligned}
$$

In (22a), each term of the objective function is represented by the variable $h_{k, n}\left(x_{k, n}\right)$ for convenience, which contains the variables $z_{k, n}$ and $f_{k, n}$. For further interpreting Eq. (22), we have:

$$
t_{k, n}\left(x_{k, n}\right)=\frac{D_{k, n}\left(x_{k, n}\right)}{W_{k, n} \log _{2}\left(1+\gamma_{k, n} \frac{P_{n}^{T}}{W_{k, n} N_{o}}\right)},
$$

where $t_{k, n}\left(x_{k, n}\right)$ is derived from Shannon's capacity formula Eq. (1), for representing the transmission duration of user $k$, when the number of transmitted video layers is $x_{k, n}$, and $D_{k, n}\left(x_{k, n}\right)$ is the total number of video bits with respect to $x_{k, n}$ that are transmitted to user $k$ in the $n$th time slot.

Applying the enumerative algorithm of [17] for solving the problem (22) suffers from a high computational complexity. Hence, we consider applying the feasible direction method of [19] to solve this challenging problem, because it can reduce the complexity of solving such non-linear programming problems. In order to invoke this method, a key step is to transform

\footnotetext{
${ }^{2}$ The minimum point of a quadratic function $y=a x^{2}+b x+c(a>0)$, is $\left(\frac{b}{-2 a}, \frac{4 a c-b^{2}}{4 a}\right)$.
} 
the optimization problem (22) into the classical feasible direction method. Let $h_{k, n}^{j}, t_{k, n}^{j}$ denote $h_{k, n}\left(x_{k, n}\right)$ and $t_{k, n}\left(x_{k, n}\right)$, respectively, where $x_{k, n}=j$, and $j \in \mathcal{J} \triangleq\{1, \ldots, L\}$. It should be noted that $h_{k, n}^{j}$ and $t_{k, n}^{j}$ are both monotonically increasing with $j$. Hence, the optimization problem (22) is rewritten as:

$$
\begin{aligned}
\text { Minimize: } & \sum_{k=1}^{K} \sum_{j=1}^{L} h_{k, n}^{j} \omega_{k, n}^{j} \\
\text { Subject to: } & \sum_{k=1}^{K} \sum_{j=1}^{L} t_{k, n}^{j} \omega_{k, n}^{j}+s=d, s \geqslant 0 \\
& \sum_{j=1}^{L} \omega_{k, n}^{j}=1, \omega_{k, n}^{j} \in\{0,1\} \\
\text { Action: } \quad & \Omega_{k, n}, \forall k,
\end{aligned}
$$

where $s$ is a slack variable, and (24c) means that every user should be served by the transmitter during time slot $n$, and we define $\Omega_{k, n}=\left(\omega_{k, n}^{1}, \omega_{k, n}^{2}, \ldots, \omega_{k, n}^{L}\right)$. The problem (24) is a constrained optimization problem, which can be solved by the classical feasible direction method.

Lemma 1. $h_{k, n}(x)$ is a convex function on $x(1 \leqslant x \leqslant L)$. Proof: See Appendix A.

The convexity of $h_{k, n}(x)$ guarantees a unique local minimum point on $[1, L]$. Hence, the feasible direction method may converge to the optimal value of (24a). According to the feasible direction method of [19], the optimal descent direction in the feasible domain is given by:

$$
\vartheta_{k^{*}, j^{*}}=\min _{k \in \mathcal{K}, j \in \mathcal{J}}\left(\frac{\delta_{k, n}^{j}}{\nu_{k, n}^{j}} \omega_{k, n}^{j}\right)=\frac{\delta_{k^{*}, n}^{j^{*}}}{\nu_{k^{*}, n}^{j^{*}}} \omega_{k^{*}, n}^{j^{*}},
$$

where $\delta_{k, n}^{j}=h_{k, n}^{j+1}-h_{k, n}^{j}, \nu_{k, n}^{j}=t_{k, n}^{j+1}-t_{k, n}^{j}$, and $\frac{\delta_{k, n}^{j}}{\nu_{k, n}^{j}}$ is the gradient of user $k$ 's utility, if the number of transmission layers increases from $j$ to $j+1$.

At the start of slot $n$, all the users are assumed to receive the base layers. Then the optimal direction $\vartheta_{k^{*}, j^{*}}$ is chosen for increasing the number of video layers of user $k^{*}$ by one. However, if the transmission duration constraint (24b) is not met, which means that the video layer of the user $k^{*}$ cannot be increased anymore, user $k^{*}$ should be removed from $\mathcal{K}$. Furthermore, it will also be removed from $\mathcal{K}$ if the number of video layers reaches the maximal value $L$. This process is repeated until there is no user left in $\mathcal{K}$, as described in Algorithm 1.

\section{Performance bound of PALS}

In this subsection, a Theorem is presented for formulating the performance bound of PALS, while minimizing the bound of (16). The parameter $V$ in (16) can be any positive constant that is greater or equal to one.

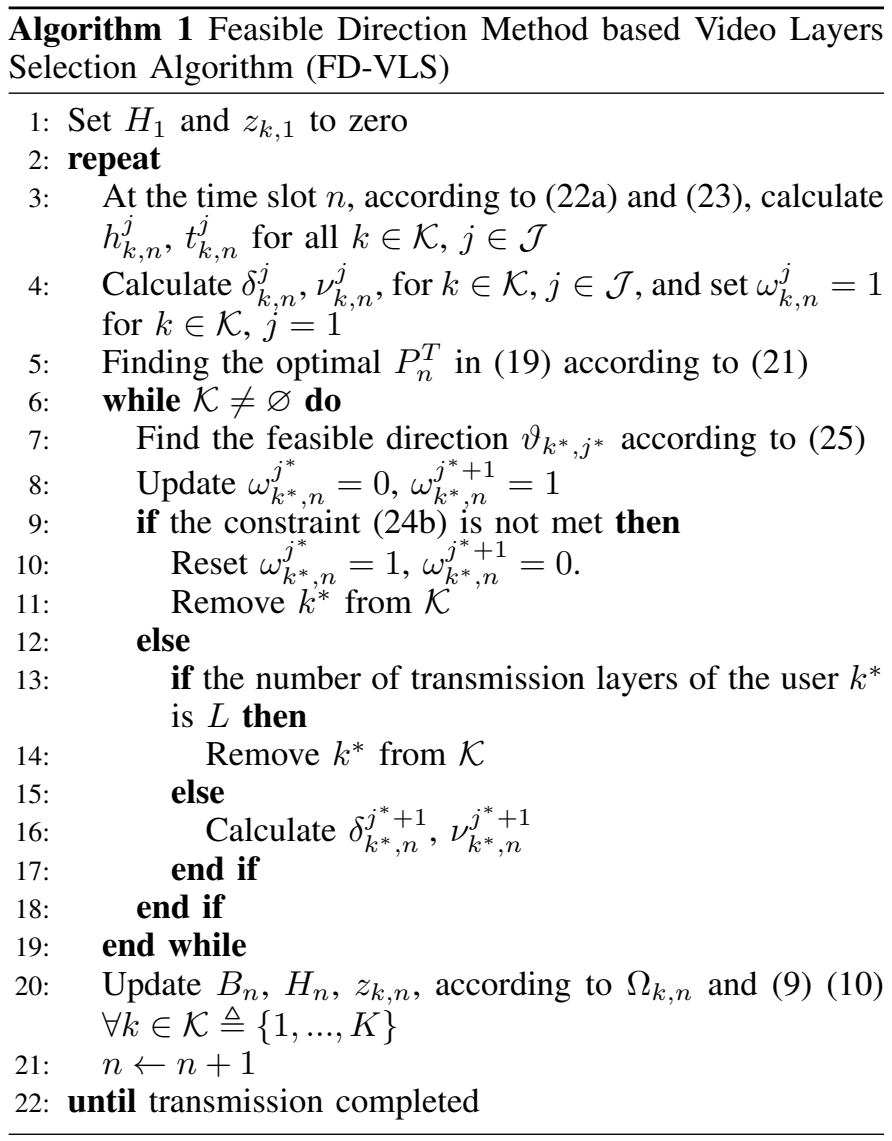

Theorem 1. The average utility $\overline{U_{P A L S}}$ obtained by the PALS strategy satisfies:

$$
\overline{U_{P A L S}}>\overline{U^{*}}-\frac{B}{V} .
$$

Here $\overline{U^{*}}$ denotes the optimal utility value in (8), and $B=$ $C_{0}+\lim _{N \rightarrow \infty} \frac{1}{N} \sum_{n=1}^{N} C_{1, n}$.

Proof: See Appendix $B$.

Inequality (26) shows that a larger $V$ can be conductive to achieving a high transmitted video quality, and when a smaller $V$ is chosen, the fluctuation of the number of video layers is more likely to be decreased (13). This conclusion is further demonstrated by the simulation results of Section V.

\section{PERFORMANCE EVALUATION}

In this section, the layer switch and playback interruption rate are chosen to be the metrics for characterizing the performance of the proposed PALS algorithm. For comparison, the heuristic algorithm ProNTO proposed in [20] is used as a benchmark, which is designed for optimizing the throughput in a proportional fair way, and without any consideration of the SVC video traffic characteristics.

\section{A. Experimental Setup}

To conduct our experiments, we set the channel bandwidth $W$ to $2 M H z$, the length of the time slot $d$ to $1 / 3 s$, and the 
TABLE I: Video trace properties

\begin{tabular}{|c|c|c|}
\hline Sequence & Elephants Dream & Big Buck Bunny \\
\hline Encoder & H.264/SVC & H.264/SVC \\
\hline Resolution & CIF $(352 \times 288)$ & CIF $(352 \times 288)$ \\
\hline GoP Pattern & G16B15 & G16B15 \\
\hline Maximal Base Layer Size (Bytes) & 55080 & 120176 \\
\hline Minimal Base Layer Size (Bytes) & 104 & 104 \\
\hline Average Base Layer Size (Bytes) & 5318.21 & 4663 \\
\hline Maximal Frame Quality (dB) & 188.13 & 188.13 \\
\hline Average Frame Quality (dB) & 42.91 & 41.57 \\
\hline
\end{tabular}

noise density to $N_{0}=4 \times 10^{-9} \mathrm{~W} / \mathrm{Hz}$. The harvested energy $E_{n}^{H}$ varies over the time slots, obeying an i.i.d. sequence, based on the Poisson distribution [17]. The channel gains between the transmitter and users obey the classic Markov process [49], [50], and the state space is discretized as $\mathscr{G}=\{$ $g_{1}=-18.82 d B, g_{2}=-13.79 d B, g_{3}=-11.23 d B, g_{4}=$ $-9.37 d B, g_{5}=-7.80 d B, g_{6}=-6.30 d B, g_{7}=-4.68 d B$, $\left.g_{8}=-2.08 d B\right\}$ similar to [51]. We used real H.264 video traces for evaluating our strategy. The video traces chosen are Elephants Dream (ED) and Big Buck Bunny (BBB) [14], and each frame of the video traces is composed of a single base layer and 6 enhancement layers. TABLE I shows the main attributes of the video sequences.

\section{B. Performance Comparison}

1) Different Average Energy Harvesting Rate: The performance differences between PALS and ProNTO were shown in Fig. 4, in conjunction with $\theta=40 \mathrm{~mJ}, \eta=0.2, V=400$, $\rho=0.9, K=8$. All the users were divided equally into two groups receiving individual SVC video sequences. In order to implement ProNTO, the time slot was partitioned into multiple equal time frames of duration $\tau$, and we set $\tau=\frac{d}{3.5 K}$. Fig. 4 shows that PALS achieves a higher quality utility, representing lower playback interruption rate and lower layer switching rate than ProNTO for both video sequences. The ProNTO method is designed mainly for optimizing the throughput, thus it is not suitable for this application scenario powered by harvested energy. Furthermore, the ProNTO method exhausted the energy harvested for transmitting as many video bits as possible, which led to a higher interruption rate and layer switching rate. From Fig. 4 (a) and (b), we can see that the utility increases while the interruption rate decreases upon increasing the average energy harvesting rate $\overline{E^{H}}$. However, beyond a certain value of $E^{H}$, the utility and interruption rate tend to become near-constant, which is because the harvested energy is sufficient for the transmitting of all the enhancement layers. The parameter $\eta$ in PALS is used for controlling the layer switching rate, which cannot be controlled in ProNTO. Fig. 4 is always around the set threshold of $\eta=0.2$, even upon increasing $\overline{E^{H}}$. Hence, $\eta$ is a parameter allowing us to control the layer switching rate.

2) Different Number of Users: To compare the performance of PALS and ProNTO when the number of users changes, we set $\theta=50 \mathrm{~mJ}, \eta=0.2, \overline{E^{H}}=129 \mathrm{~mJ}, V=400$, and the total number of users $K$ was varying from 3 to 20 . Upon increment $K$ in PALS, the amount of reserved energy

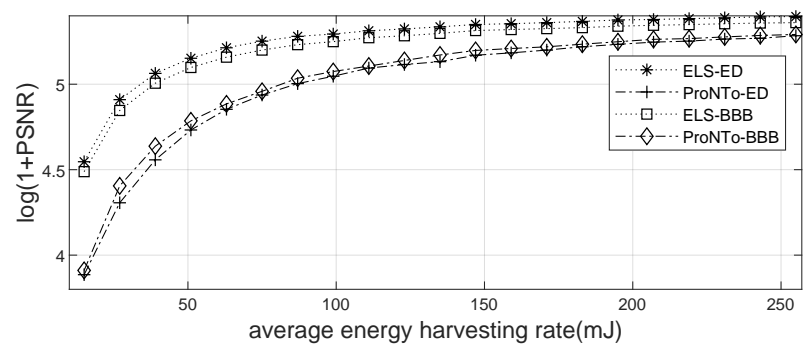

(a) Utility.

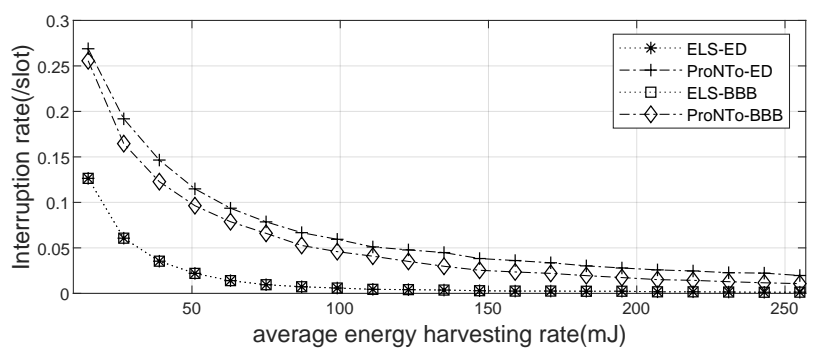

(b) Interruption rate

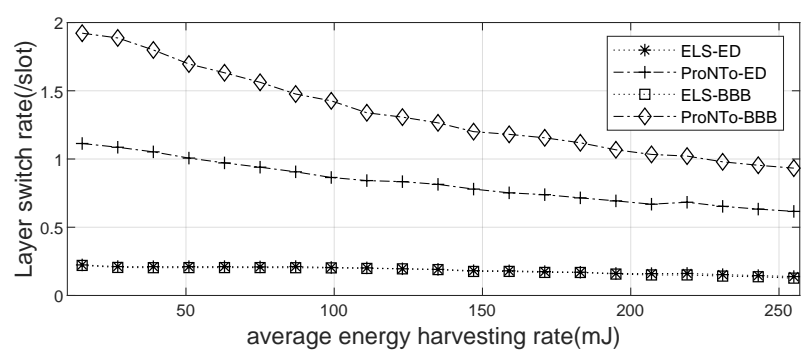

(c) Layer switching rate.

Fig. 4: Performance comparison for different energy harvesting rates.

is no longer sufficient for all the base layers' transmission. This increases the interruption rate and reduces the utility upon increasing $K$, but the layer switching rate is still no higher than the set threshold of $\eta=0.2$ (Fig. 5). Compared to ProNTO, which does not reserve energy for the forthcoming time slots, the utility of PALS decreases while the interruption rate increases less sharply than that of ProNTO upon increasing the number of users. Furthermore, without any video layer fluctuation limitations, the layer switching rate of ProNTO is much higher than that of PALS.

\section{Parameter Sensitivity Analysis}

1) Parameter Sensitivity of $\theta$ : Given $K=8, \overline{E^{H}}=129 \mathrm{~mJ}$, $\eta=0.1, V=300$, this experiment was conducted to investigate the system performance sensitivity wrt $\theta$. The utility decreases upon increasing $\theta$, which is because a large value of $\theta$ means that more energy should be reserved in the battery. Thus, less enhancement layers are transmitted, as illustrated in Fig. 6. Furthermore, playback interruptions are less likely to 

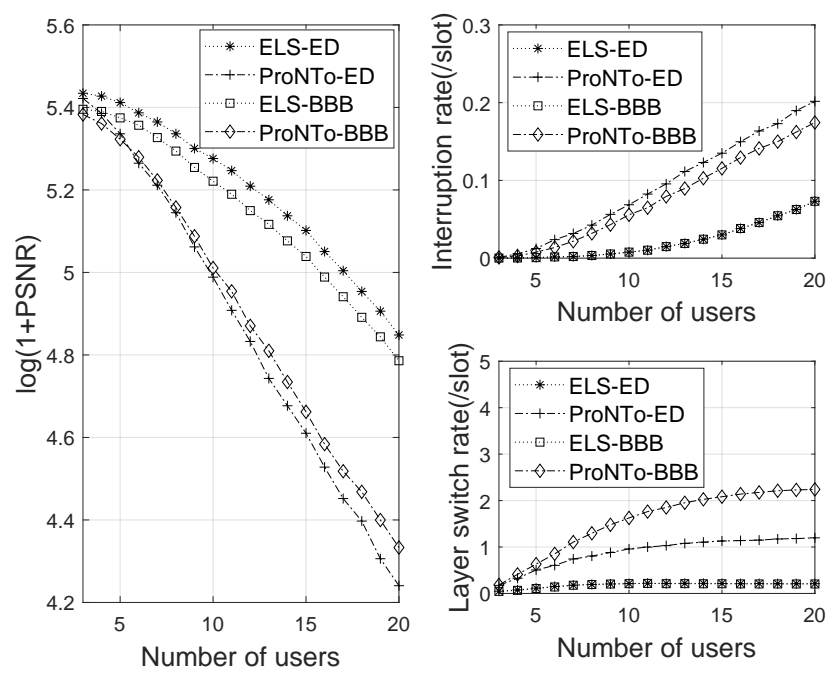

Fig. 5: Performance comparison for different number of users.
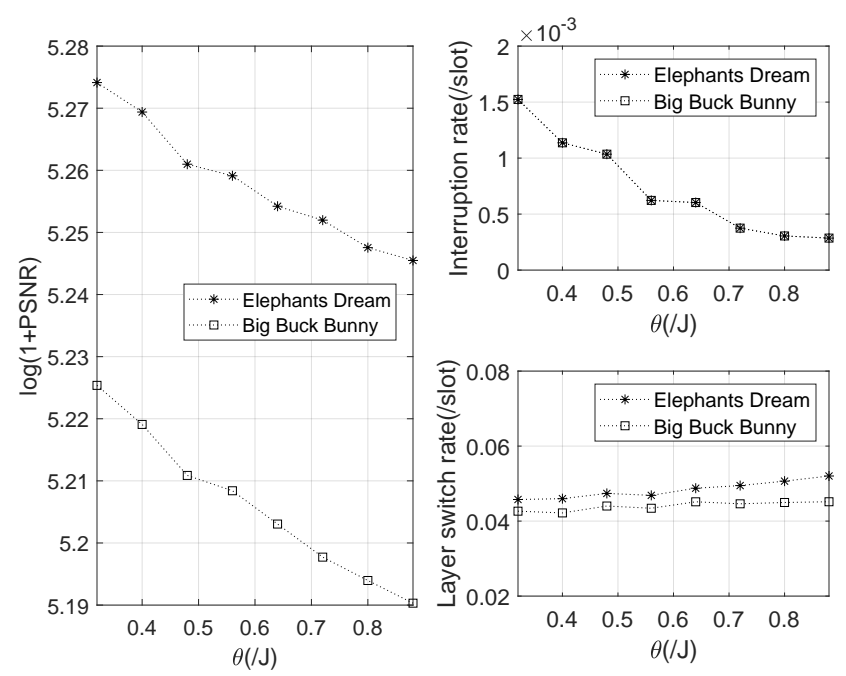

Fig. 6: The system performance sensitivity to different value of $\theta$.

occur, since the reserved energy is sufficient. Interestingly, the layer switching rate is always below the threshold of $\eta=0.1$ as shown in Fig. 6, and it stabilizes even for an increased $\theta$. The reason for this is that we imposed an explicit constraint on the layer switching rate, constraining it to be lower than $\eta$ in our optimization model.

2) Parameter Sensitivity of $\eta$ : This experiment was conducted for investigating the system performance sensitivity to $\eta$, and the parameters were set to $K=8, \overline{E^{H}}=129 \mathrm{~mJ}$, $\theta=60 m J, V=50$, and $\eta$ varying from 0.1 to 1 with a step size of 0.1. The experimental results were shown in Fig. 7 , which demonstrate that the layer switching rate increases
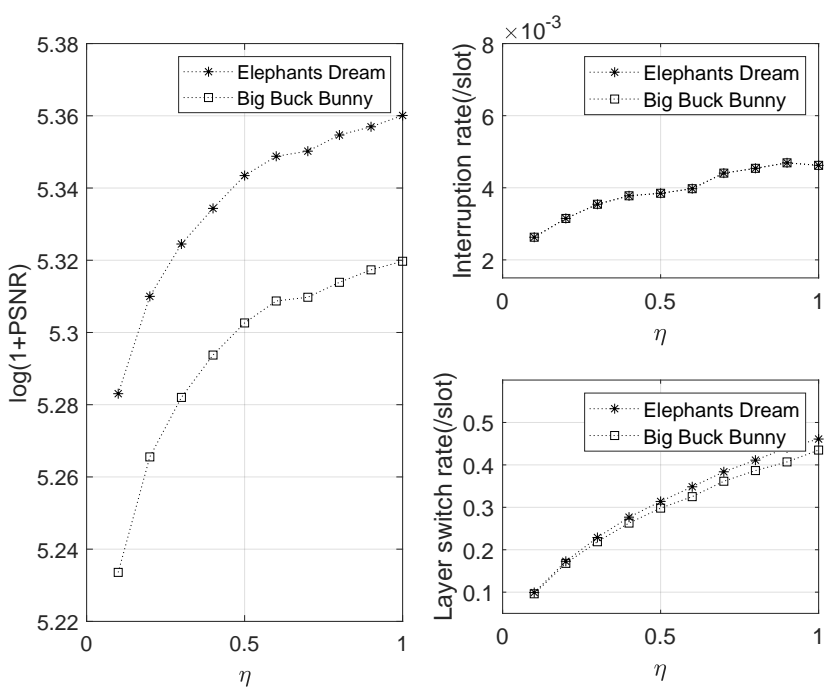

Fig. 7: The system performance sensitivity to different value of $\eta$.

steadily, as $\eta$ becomes larger. By increasing the threshold $\eta$, more enhancement layers are likely to be transmitted, and thus the value of the utility is improved. However, this will lead to more energy being consumed and less energy reserved for the forthcoming slots. As a result, the interruption rate is increased upon increasing $\eta$. In different video services, the threshold value of $\eta$ can be different. In general, $\eta=0.2$ may be advocated to maintain a relatively low layering switching rate and interruption rate.

3) Parameter Sensitivity of $V$ : The experiment in this section was conducted for investigating the sensitivity of PALS to the factor $V$. The parameters were set as: $K=8$, $\overline{E^{H}}=129 \mathrm{~mJ}, \theta=40 \mathrm{~mJ}, \eta=0.2$, and $V$ grows from 50 to 600 at a step size of 50 . According to the analysis in Section IV-A, a larger value of $V$ is conductive to a higher utility of PALS, as demonstrated in Fig. 8. Nevertheless, upon increasing $V$, the growth of the utility slows down. Observe from (17) that upon increasing $V$, the proposed algorithm tends to increase the utility by choosing more video layers for transmission, which, in turn, increases the layer switching rate and consumes more energy for video transmission. Then, the playback interruption rate increases, since the reserved energy is insufficient. This is the reason for the slow growth of the utility. The results demonstrate that a tradeoff between the playback smoothness and system utility representing the video distortion can be struck by adjusting $V$. According to Fig. 8, the value of $V$ should be no more than 500 to maintain a relatively low layer switching rate of less than 0.2 .

\section{CONCLUSION}

The multi-user scalable video transmission problem of EH aided wireless communication systems was studied. By aiming for improving the received video quality while maintaining fairness amongst the users in the face of the random properties 

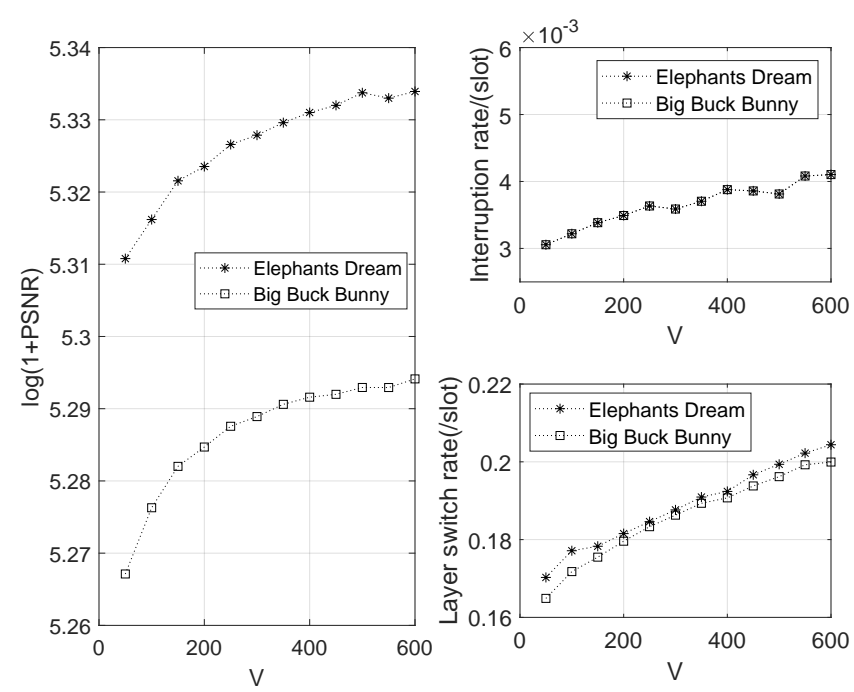

Fig. 8: The system performance sensitivity to different value of $V$.

of both energy harvesting and of the channel quality, we have formulated the CUFM problem associated with proportional fairness and playback smoothness guarantees. By introducing the classical Lyapunov drift and optimization technique, the CUFM problem was decomposed into a pair of independent sub-problems:the power allocation problem and layer selection problem. We also employed the feasible direction method to solve the layer selection problem and reduced the computational complexity order to $O(K L)$. Furthermore, we have derived the theoretical performance bound for characterizing the proposed solution. Our simulation results also show that the PALS algorithm provides a higher performance than the ProNTO benchmark algorithm [20].

As our future work, inspired by [52]-[55], the SVC video streaming service will be considered in the context of wireless mesh networks, where the optimal layer selection and routing problems should be jointly tackled for improving the QoE of the end users.

\section{APPENDIX A}

\section{PROOF OF LEMMA 1}

Proof: For (22a), the first term $\zeta(x)=$ $z_{k, n}\left(\left|x-x_{k, n-1}\right|-\eta\right)$ is a convex function. The last part of (22a) is:

$$
\psi(x)=-V \log _{2}\left[Q_{k, n}(x)\right] .
$$

According to the properties of convex functions, if $Q_{k, n}(x)$ is a concave function, $\psi(x)$ can be proved to be convex. Please refer to Fig. 9 of [14] to observe that the average frame PSNR $Q_{k, n}(x)$ is a concave function around the average transmitted video layers $(1 \leq x \leq L)$. Thus, the convexity of $h_{k, n}(x)=\zeta(x)+\psi(x)$ is demonstrated.

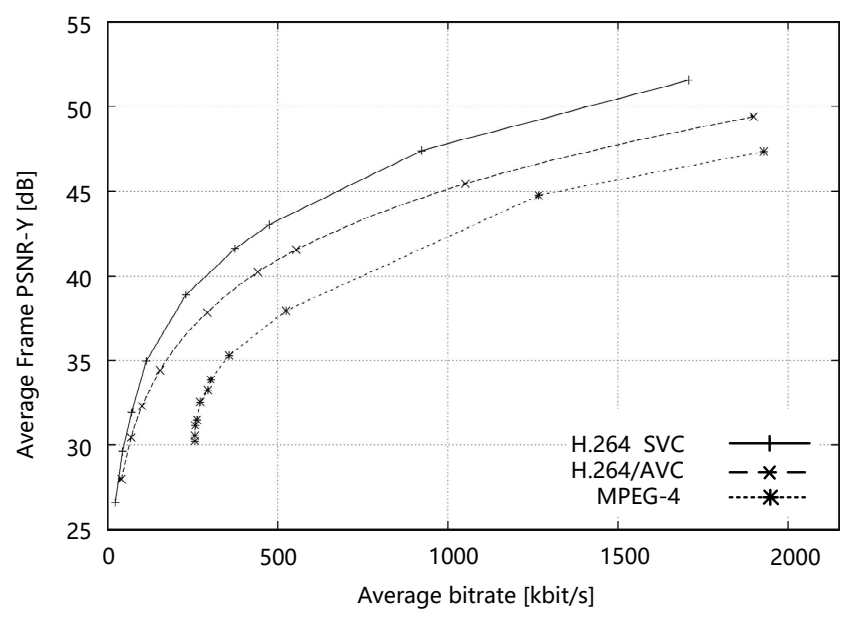

Fig. 9: Rate-distortion (RD) plots for the Big Buck Bunny video traces encoded in MPEG-4, H.264/AVC, and H.264 SVC.

\section{APPENDIX B \\ PROOF OF THEOREM 1}

Proof: The proposed PALS algorithm tries to minimize the right side of (16), and hence we have the following inequality

$$
\begin{aligned}
\Delta\left(\boldsymbol{\Theta}_{n}\right)- & V \mathbb{E}\left\{U_{P A L S}\left(X_{n}\right) \mid \Theta_{n}\right\} \\
\leqslant & \min _{X_{n}}\left\{C_{0}+C_{1, n}\right. \\
& +\frac{1}{2} \mathbb{E}\left\{\left(E_{n}^{t}\right)^{2}\right\}+\left(B_{n}-H_{n}\right) \mathbb{E}\left(E_{n}^{H}-E_{n}^{T}\right) \\
& \left.+\sum_{k=1}^{K} \mathbb{E}\left\{z_{k, n}\left(f_{k, n}-\eta\right)\right\}-V \mathbb{E}\left\{U_{P A L S}\left(X_{n}\right)\right\}\right\} .
\end{aligned}
$$

Here the left part is the Lyapunov "drift-plus-penalty" term. According to [47], the optimal system utility value can be achieved by a stationary and randomized $\omega$-only strategy, while the constraint (4) and (5) are also satisfied. The stochastic nature of the harvested energy is the single factor relevant to the stationary $\omega$-only strategy. Hence substituting the optimal $\omega$-only strategy into the PALS of (28), and exploiting the fact that $\mathbb{E}\left\{U^{*}\right\}=\overline{U^{*}}$, we have

$$
\Delta\left(\boldsymbol{\Theta}_{n}\right)-V \mathbb{E}\left\{U_{P A L S}\left(X_{n}\right) \mid \Theta_{n}\right\} \leqslant C_{0}+C_{1, n}-V \overline{U^{*}}
$$

Now taking the expectations on both sides of (29), we get the following inequality:

$$
\begin{array}{r}
\mathbb{E}\left\{L\left(\boldsymbol{\Theta}_{n+1}\right)\right\}-\mathbb{E}\left\{L\left(\boldsymbol{\Theta}_{n}\right)\right\}-V \mathbb{E}\left\{U_{P A L S}\left(X_{n}\right) \mid \boldsymbol{\Theta}_{n}\right\} \\
\leqslant C_{0}+C_{1, n}-V \overline{U^{*}}
\end{array}
$$


Summing (30) over $n=0,1, \ldots, N-1$ results in:

$$
\begin{aligned}
& \mathbb{E}\{L(N)\}-\mathbb{E}\{L(0)\}- V \sum_{n=0}^{N-1} \mathbb{E}\left\{U\left(X_{n}\right)\right\} \\
& \leqslant N C_{0}+\sum_{n=0}^{N-1} C_{1, n}-N V \overline{U^{*}}
\end{aligned}
$$

Upon rearranging the terms, due to the fact that $L(N)>0$ and $L(0)=0$, and then dividing both sides by $N V$, we have

$$
\begin{aligned}
& \lim _{N \rightarrow \infty} \frac{1}{N} \sum_{n=0}^{N-1} \mathbb{E}\left\{U\left(X_{n}\right)\right\} \\
& \geqslant \overline{U^{*}}-\frac{C_{0}+\lim _{N \rightarrow \infty} \frac{1}{N} \sum_{n=0}^{N-1} C_{1, n}}{V} .
\end{aligned}
$$

This proves Theorem 1.

\section{ACKNOWLEDGMENT}

This work was supported by National Natural Science of China(No. 61573329), Equipment pre-Research Project (No. 6141B0801010a) and Youth Innovation Promotion Assocation CAS.

\section{REFERENCES}

[1] IDTechEx, "Market for energy harvesting to reach $\$ 2.6$ billion by 2024," https://www.idtechex.com/research/articles/market-for-energyharvesting-to-reach-2-6-billion-by-2024-00007051.asp, accessed Nov. 06, 2014.

[2] H. Zhang, S. Huang, C. Jiang, K. Long, V. C. M. Leung, and H. V. Poor, "Energy efficient user association and power allocation in millimeterwave-based ultra dense networks with energy harvesting base stations,' IEEE J. Sel. Areas Commun., vol. 35, no. 9, pp. 1936-1947, Sep. 2017.

[3] Y. Zhang, D. Zhang, L. Pang, M. Chi, Y. Li, G. Ren, and J. Li, "Power optimization for massive MIMO systems with hybrid energy harvesting transmitter," IEEE Trans. Veh. Technol., vol. 67, no. 10, pp. 10039 10043 , Oct. 2018.

[4] Y. Guo, Q. Yang, F. R. Yu, and V. C. M. Leung, "Cache-enabled adaptive video streaming over vehicular networks: A dynamic approach," IEEE Trans. Veh. Technol., vol. 67, no. 6, pp. 5445-5459, Jun. 2018.

[5] J. Yang, P. Si, Z. Wang, X. Jiang, and L. Hanzo, "Dynamic resource allocation and layer selection for scalable video streaming in femtocell networks: A twin-time-scale approach," IEEE Trans. Commun., vol. 66, no. 8, pp. 3455-3470, Aug. 2018.

[6] J. Yang, B. Yang, S. Chen, Y. Zhang, Y. Zhang, and L. Hanzo, "Dynamic resource allocation for streaming scalable videos in SDN-aided dense small-cell networks," IEEE Trans. Commun., vol. 67, no. 3, pp. 2114 2129, Mar. 2019.

[7] L. Sun, H. Shan, A. Huang, L. Cai, and H. He, "Channel allocation for adaptive video streaming in vehicular networks," IEEE Trans. Veh. Technol., vol. 66, no. 1, pp. 734-747, Jan. 2017.

[8] C. Inc., "Cisco visual networking index: Forecast and methodology, 2016-2021," https://www.cisco.com/c/en/us/solutions/collateral/serviceprovider/visual-networking-index-vni/complete-white-paper-c11481360.html, accessed Sep. 15, 2017.

[9] S. Gupta, R. Zhang, and L. Hanzo, "Throughput maximization for a buffer-aided successive relaying network employing energy harvesting," IEEE Trans. Veh. Technol., vol. 65, no. 8, pp. 6758-6765, Aug. 2016.

[10] C. Huang, R. Zhang, and S. Cui, "Throughput maximization for the Gaussian relay channel with energy harvesting constraints," IEEE $J$. Sel. Areas Commun., vol. 31, no. 8, pp. 1469-1479, Aug. 2013.
[11] S. Lohani, E. Hossain, and V. K. Bhargava, "Joint resource allocation and dynamic activation of energy harvesting small cells in OFDMA HetNets," IEEE Trans. Wireless Commun., vol. 17, no. 3, pp. 17681783, Mar. 2018.

[12] J. Xie, W. Cai, L. Deng, and J. Yang, "QoS-constrained transmission policy in hybrid energy supply wireless communication system," in VTC Spring, Jun. 2017, pp. 1-6.

[13] Z. Li, Y. Gao, P. Li, B. A. Salihu, L. Sang, and D. Yang, "Throughput analysis of an energy harvesting multichannel system under delay and energy storage constraints," IEEE Trans. Veh. Technol., vol. 66, no. 9, pp. 7818-7832, Sep. 2017.

[14] P. Seeling and M. Reisslein, "Video transport evaluation with H.264 video traces," IEEE Commun. Surveys Tuts., vol. 14, no. 4, pp. 11421165, 2012.

[15] G. Giambene, S. Kota, and P. Pillai, "Satellite-5G integration: A network perspective," IEEE Netw., vol. 32, no. 5, pp. 25-31, Sep. 2018.

[16] S. Zhou, G. Wang, S. Zhang, Z. Niu, and X. S. Shen, "Bidirectional mission offloading for agile space-air-ground integrated networks," IEEE Wireless Commun. Mag., vol. 26, no. 2, pp. 38-45, Apr. 2019.

[17] X. Wu, J. Yang, Y. Ran, and H. Xi, "Adaptive scalable video transmission strategy in energy harvesting communication system," IEEE Trans. Multimedia, vol. 17, no. 12, pp. 2345-2353, Dec. 2015.

[18] J. Yang, W. Cai, Y. Ran, H. Xi, and L. Hanzo, "Online measurementbased adaptive scalable video transmission in energy harvesting aided wireless systems," IEEE Trans. Veh. Technol., vol. 66, no. 7, pp. 62316245, Jul. 2017.

[19] N. S. Rau, Constrained Nonlinear Optimization. IEEE, 2003. [Online]. Available: https://ieeexplore.ieee.org/document/5263486

[20] N. Tekbiyik, T. Girici, E. Uysal-Biyikoglu, and K. Leblebicioglu, "Proportional fair resource allocation on an energy harvesting downlink," IEEE Trans. Wireless Commun., vol. 12, no. 4, pp. 1699-1711, Apr. 2013.

[21] X. Lu, P. Wang, D. Niyato, D. I. Kim, and Z. Han, "Wireless networks with RF energy harvesting: A contemporary survey," IEEE Commun. Surveys Tuts., vol. 17, no. 2, pp. 757-789, Secondquarter 2015.

[22] X. Jiang, X. Wang, H. Xi, and F. Liu, "Centralized optimization for DecPOMDPs under the expected average reward criterion," IEEE Trans. Autom. Control, vol. 62, no. 11, pp. 6032-6038, Nov. 2017.

[23] J. Gong, S. Zhou, and Z. Niu, "Optimal power allocation for energy harvesting and power grid coexisting wireless communication systems," IEEE Trans. Commun., vol. 61, no. 7, pp. 3040-3049, Jul. 2013.

[24] J. Yang and S. Ulukus, "Optimal packet scheduling in an energy harvesting communication system," IEEE Trans. Commun., vol. 60, no. 1, pp. 220-230, Jan. 2012.

[25] D. Mishra and S. De, "i2RES: Integrated information relay and energy supply assisted RF harvesting communication," IEEE Trans. Commun., vol. 65, no. 3, pp. 1274-1288, Mar. 2017.

[26] V. D. Nguyen, T. Q. Duong, H. D. Tuan, O. S. Shin, and H. V. Poor, "Spectral and energy efficiencies in full-duplex wireless information and power transfer," IEEE Trans. Commun., vol. 65, no. 5, pp. 2220-2233, May. 2017.

[27] J. Yang, Q. Yang, K. S. Kwak, and R. R. Rao, "Power-delay tradeoff in wireless powered communication networks," IEEE Trans. Veh. Technol., vol. 66, no. 4, pp. 3280-3292, Apr. 2017.

[28] L. P. Qian, G. Feng, and V. C. M. Leung, "Optimal transmission policies for relay communication networks with ambient energy harvesting relays," IEEE J. Sel. Areas Commun., vol. 34, no. 12, pp. 3754-3768, Dec. 2016.

[29] X. Jiang, X. Wang, and H. Xi, "Finding optimal polices for wideband spectrum sensing based on constrained POMDP framework," IEEE Trans. Wireless Commun., vol. 16, no. 8, pp. 5311-5324, Aug. 2017.

[30] D. Zhang, Z. Chen, J. Ren, N. Zhang, M. K. Awad, H. Zhou, and X. S. Shen, "Energy-harvesting-aided spectrum sensing and data transmission in heterogeneous cognitive radio sensor network," IEEE Trans. Veh. Technol., vol. 66, no. 1, pp. 831-843, Jan. 2017. 
[31] Y. Li, M. Sheng, Y. Sun, and Y. Shi, "Joint optimization of bs operation, user association, subcarrier assignment, and power allocation for energy-efficient HetNets," IEEE J. Sel. Areas Commun., vol. 34, no. 12 , pp. 3339-3353, Dec. 2016.

[32] D. Altinel and G. K. Kurt, "Finite-state markov channel based modeling of RF energy harvesting systems," IEEE Trans. Veh. Technol., vol. 67 , no. 2, pp. 1713-1725, Feb. 2018.

[33] Y. Li, T. Jiang, K. Luo, and S. Mao, "Green heterogeneous cloud radio access networks: Potential techniques, performance trade-offs, and challenges," IEEE Commun. Mag., vol. 55, no. 11, pp. 33-39, Nov. 2017.

[34] S. Kim, G. Suk, J. Lee, and C. Chae, "Qoe-aware scalable video transmission in MIMO systems," IEEE Commun. Mag., vol. 55, no. 8 , pp. 196-203, Aug. 2017

[35] X. Chen, J. N. Hwang, C. N. Lee, and S. I. Chen, "A near optimal QoE-driven power allocation scheme for scalable video transmissions over MIMO systems," IEEE J. Sel. Top. Sign. Proces., vol. 9, no. 1, pp. 76-88, Feb. 2015.

[36] X. Chen, J. N. Hwang, J. A. Ritcey, C. N. Lee, and F. M. Yeh, "Quality-driven joint rate and power adaptation for scalable video transmissions over MIMO systems," IEEE Trans. Circuits Syst. Video Technol., vol. 27, no. 2, pp. 366-379, Feb. 2017.

[37] S. Cical and V. Tralli, "Distortion-fair cross-layer resource allocation for scalable video transmission in OFDMA wireless networks," IEEE Trans. Multimedia, vol. 16, no. 3, pp. 848-863, Apr. 2014.

[38] S. Chen, J. Yang, Y. Ran, and E. Yang, "Adaptive layer switching algorithm based on buffer underflow probability for scalable video streaming over wireless networks," IEEE Trans. Circuits Syst. Video Technol., vol. 26, no. 6, pp. 1146-1160, Jun. 2016.

[39] H. Zhou, Y. Ji, and B. Zhao, "Tabu-search-based metaheuristic resourceallocation algorithm for SVC multicast over wireless relay networks," IEEE Trans. Veh. Technol., vol. 64, no. 1, pp. 236-247, Jan. 2015.

[40] E. Yaacoub, F. Filali, and A. Abu-Dayya, "QoE enhancement of SVC video streaming over vehicular networks using cooperative LTE/802.11p communications," IEEE J. Sel. Top. Sign. Proces., vol. 9, no. 1, pp. 3749, Feb. 2015.

[41] S. A. Hosseini, Z. Lu, G. de Veciana, and S. S. Panwar, "SVC-based multi-user streamloading for wireless networks," IEEE J. Sel. Areas Commun., vol. 34, no. 8, pp. 2185-2197, Aug. 2016.

[42] A. Ahmad, N. Ul Hassan, M. Assaad, and H. Tembine, "Joint power control and rate adaptation for video streaming in wireless networks with time-varying interference," IEEE Trans. Veh. Technol., vol. 65, no. 8, pp. 6315-6329, Aug. 2016.

[43] Y. Huo, C. Hellge, T. Wiegand, and L. Hanzo, "A tutorial and review on inter-layer FEC coded layered video streaming," IEEE Commun. Surveys Tuts., vol. 17, no. 2, pp. 1166-1207, Secondquarter 2015.

[44] L. Hanzo, P. Cherriman, and J. Streit, Video Compression and Communications: From Basics to H.261, H.263, H.264, MPEG4 for DVB and HSDPA-Style Adaptive Turbo-Transceivers, 2nd ed. Wiley-IEEE Press, 2007.

[45] M. Gorlatova, A. Wallwater, and G. Zussman, "Networking low-power energy harvesting devices: Measurements and algorithms," IEEE Trans. Mobile Comput., vol. 12, no. 9, pp. 1853-1865, Sep. 2013

[46] R. S. Liu, P. Sinha, and C. E. Koksal, "Joint energy management and resource allocation in rechargeable sensor networks," in IEEE INFOCOM, Mar. 2010, pp. 1-9.

[47] M. J. Neely, Stochastic Network Optimization with Application to Communication and Queueing Systems. Morgan and Claypool Publishers, 2010 .

[48] E. S. Gardner, "Exponential smoothing: The state of the art," J FORECASTING, vol. 4, no. 1, pp. 1-28, 1985.

[49] S. Tsai, "Markov characterization of the HF channel," IEEE Trans. Commun. Technol., vol. 17, no. 1, pp. 24-32, Feb. 1969.

[50] H. S. Wang and P.-C. Chang, "On verifying the first-order Markovian assumption for a Rayleigh fading channel model," IEEE Trans. Veh. Technol., vol. 45, no. 2, pp. 353-357, May. 1996.

[51] N. Mastronarde and M. van der Schaar, "Joint physical-layer and system-level power management for delay-sensitive wireless communications," IEEE Trans. Mobile Comput., vol. 12, no. 4, pp. 694-709, Apr. 2013.

[52] C. Dong, L. Yang, and L. Hanzo, "Performance analysis of multihopdiversity-aided multihop links," IEEE Trans. Veh. Technol., vol. 61, no. 6, pp. 2504-2516, Jul. 2012.

[53] D. Chen, L. Yang, and L. Hanzo, "Multi-hop diversity aided multi-hop communications: A cumulative distribution function aware approach," IEEE Trans. Commun., vol. 61, no. 11, pp. 4486-4499, Nov. 2013.

[54] J. Hu, K. Yang, G. Wen, and L. Hanzo, "Integrated data and energy communication network: A comprehensive survey," IEEE Commun. Surveys Tuts., vol. 20, no. 4, pp. 3169-3219, Fourthquarter 2018.

[55] C. Dong, L. Li, B. Zhang, L. Yang, and L. Hanzo, "Energy dissipation versus delay tradeoffs in a buffer-aided two-hop link," IEEE Trans. Veh. Technol., vol. 65, no. 10, pp. 8060-8071, Oct. 2016. 